\title{
Alcool et neurochimie
}

\begin{abstract}
Les effets de l'alcool sur le système nerveux sont faciles à constater mais difficiles à exprimer en termes moléculaires. Membranes cellulaires, neurotransmetteurs, neuropeptides, canaux ioniques subissent des altérations qui diffèrent selon que l'intoxication est aiguë ou chronique.
\end{abstract}

\section{Marc Ledig \\ Chargé de recherche au Cnrs}

\section{Paul Mandel \\ Professeur honoraire à la faculté de médecine de Stras- bourg}

\section{RÉFÉRENCES}

1. Le Bourhis B. Sur l'établissement de la dépendance des rats à l'égard de l'alcool. Physiol Behav 1977 ; 18 : 475-8.

2. Ledig M, M'Paria JR, Louis JC, Fried R, Mandel P. Effect of ethanol on superoxide dismutase activity in cultured neural cells. Neurochem Res 1980; 5 : 1155-62.

\section{ADRESSE}

M. Ledig, P. Mandel : centre de neurochimie du Cnrs, 5, rue Blaise-Pascal, 67084 Stras- 'alcool, comme certains anesthésiques, exerce un effet biphasique. A faible dose, il est euphorisant, voire stimulant des facultés intellectuelles, alors qu'audelà d'un certain seuil, variable d'un individu à un autre, il produit un effet dépresseur. Les mécanismes moléculaires complexes qui soustendent ces phénomènes ne sont que partiellement élucidés.

Souvent ils ne sont pas spécifiques d'un organe ou d'un tissu. C'est pourquoi on pourra trouver ici la description de phénomènes mentionnés également dans d'autres articles de ce numéro. Ce travail tente de cerner ce qui est propre aux rapports entre l'intoxication alcoolique et le système nerveux.

La difficulté majeure à laquelle se heurte l'étude expérimentale est l'absence d'un modèle d'étude. Contrairement à l'homme, les animaux, à l'exception du chimpanzé, refusent généralement de boire de l'alcool et ne deviennent jamais spontanément alcooliques. Il existe cependant des souches de souris et de rats qui boivent plus volontiers de l'alcool que d'autres, mais les quantités d'alcool absorbées demeurent faibles. Il faut donc faire appel à un certain nombre d'artifices pour administrer de l'alcool à des animaux : injections intrapéritonéales ou intraveineuses, intubation gastrique ou maintien des animaux dans une atmosphère contenant des vapeurs d'alcool [1]. On est loin de la situation de consommation spontanée excessive de l'homme. Il cxiste cependant un modèle expérimental intéressant : les cultures de cellules en présence d'alcool, mais une telle approche a évidemment une portée limitée [2]

In vivo les effets de l'alcool vont de pair avec sa concentration sanguine qui décroît plus ou moins vite selon les espèces et les souches, essentiellement à la suite de son catabolisme au niveau du foie. Nous avons cependant montré que le cerveau était également équipé pour dégrader l'alcool, mais à un degré moindre que le foie [3]. De nombreuses études et des recherches cliniques ont mis en relief des altérations comportementales consécutives à la consommation d'alcool : déficit de l'apprentissage, de la mémorisation et des aptitudes conceptuelles. Dans les cas les plus graves, on observe diverses formes d'encéphalopathies. Parmi celles-ci se place le syndrome fotal 


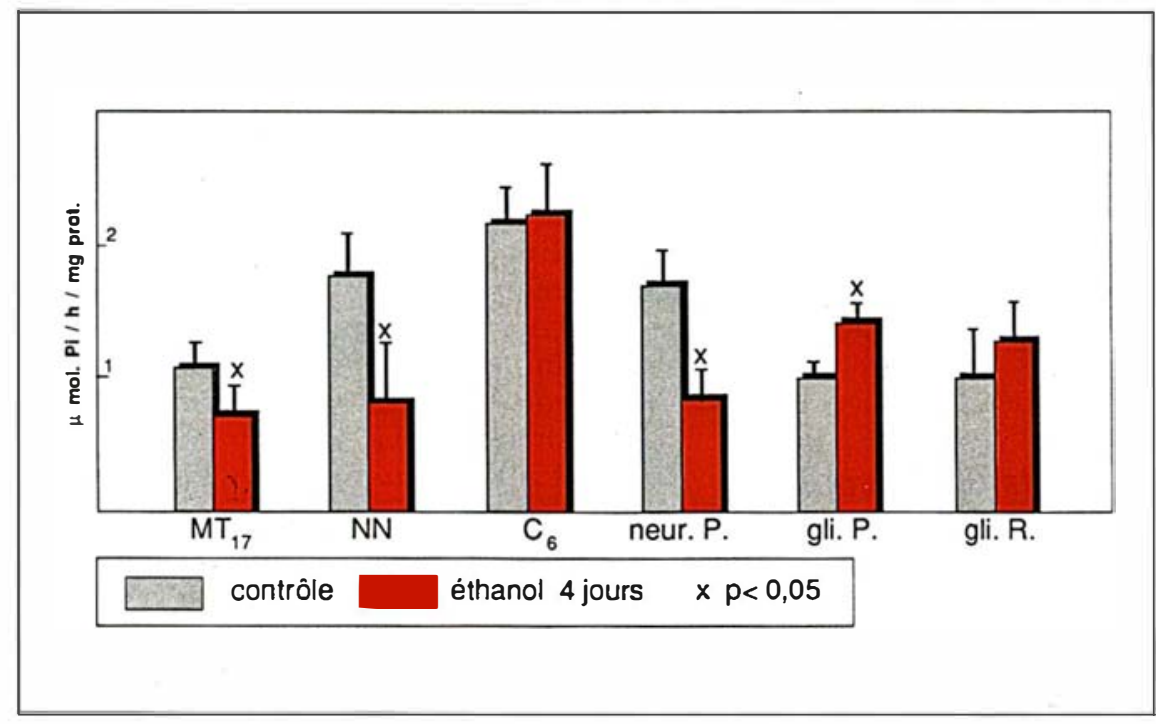

Figure 1. Activité de l'ATPase $\left(\mathrm{Na}^{+}, \mathrm{K}^{+}\right)$de cellules nerveuses en culture (D) et traitées pendant 4 jours avec de l'éthanol $100 \mathrm{mM}$ (D) . MT17 = lignée neuronale issue d'un neuroblastome, $N N=$ lignée d'astrocytes transformés C6 = lignée issue d'un gliome, neur. $P .=$ neurones de poulet, gli. $P .=$ cellules gliales de poulet, gli. $R$. = cellules gliales de rat. $x=p<0,05$.

alcoolique, conséquence de l'effet tératogène de l'éthanol in utero.

La consommation chronique d'alcool conduit à un état de dépendance. Les facteurs qui conditionnent la dépendance sont complexes; les quantités absorbées, la périodicité et la durée de la consommation sont déterminantes [4]. La privation d'alcool provoque un syndrome de sevrage qui s'accompagne d'un état d'hyperexcitabilité et parfois de crises convulsives que l'on peut prévenir par l'administration de GABA* mimétiques. L'intervention d'altérations de la neurotransmission GABAergique et sérotoninergique dans le syndrome de sevrage a été suggérée.

Des facteurs génétiques interviennent très probablement dans la prédisposition à l'alcoolisme. Nous avons montré récemment, chez l'homme, que le chromosome 6 semble être plus particulièrement impliqué dans le déterminisme génétique de l'alcoolisme [5]. Des facteurs immulogiques semblent être associés à des facteurs génétiques [6].

Les états dépressifs ou anxieux ainsi que les stress créent également des terrains favorables au développement de l'alcoolisme. Afin de mieux comprendre les effets aigus ou chroni-

* GABA : acide $\gamma$-aminobutyrique.

$\mathrm{m} / \mathrm{s} n^{\circ} 6$ vol. 4 , juin 88 quant cette technique à des membranes synaptosomales isolées de rats ayant reçu une dose unique d'alcool on constate une augmentation de la fluidité membranaire alors qu'après un traitement chronique on observe une diminution de celle-ci. On a suggéré que ce phénomène pourrait être impliqué dans la tolérance acquise [8]. Les études concernant les effets de l'alcool sur la composition lipidique des structures membranaires ont fait apparaître des modifications du rapport lipides neutres phospholipides acides [9] dues essentiellement à une augmentation du taux des polyphosphoinositides [10]. Lorsque l'on incube des membranes synaptiques en présence d'éthanol, on constate une diminution de l'activité ATPasique $\left(\mathrm{Na}^{+}, \mathrm{K}^{+}\right)[11]$. Il en est de même lors de l'administration aiguë d'éthanol à des rats; à l'opposé, le traitement chronique conduit à une augmentation de cette activité enzymatique dans le cerveau. L'activité ATPasique $\left(\mathrm{Mg}^{++}\right)$située essentiellement à l'intérieur des cellules nerveuses ne paraît pas affectée par l'alcool. Nous avons montré qu'il existait des différences entre les cellules neuronales et gliales quant à la réponse de l'activité ATPasique $\left(\mathrm{Na}^{+}, \mathrm{K}^{+}\right)$à la présence d'alcool (figure 1). Il en est de même pour l'activité des ATPases ecto-membranaires, en particulier l'activité ectoATPase $\left(\mathrm{Ca}^{++}\right)$. Les différences observées entre les cellules neuronales et gliales pourraient être en rapport avec la composition lipidique et lipoprotéique spécifique de leurs membranes plasmiques [12]. En effet, l'activité de ces enzymes dépend essentiellement de leur environnement lipidique. Étant donné que l'alcool modifie les lipides membranaires, il s'ensuit un changement de l'activité de ces enzymes.

On sait par ailleurs que le métabolisme de l'alcool peut engendrer des radicaux libres parallèlement aux modifications du rapport $\mathrm{NAD}^{+}$/ NADH [13]. En effet lorsque ce rapport est modifié, l'acétaldéhyde peut être oxydé en acétate par la xanthine oxydase produisant en même temps des radicaux superoxydes cytotoxiques qui sont transformés en peroxyde par la superoxyde dismu- 


\section{RÉFÉRENCES}

3. Mandel P, Ledig M, M'Paria JR. Ethanol and neuronal metabolism. Pharmacol Bio chem Behav 1980 ; 13 : 175-82.

4. Le Magnen J. L'alcoolisme. La Recherche $1980 ; 11: 1183-9$.

5. Ledig M, Doffoel M, Tongio MM, et al. Frequencies of Glyoxalase I, phenotypes as biological markers in chronic alcoholism. Alcohol 1986; 3 : 11-4.

6. Doffoel M, Tongio MM, Gut JP, et al Relationship between thirty-four HLA-A, B and DR antigens and three serological markers of viral infections in alcoholic cirrhosis. Hepatology 1986 ; 6 : 457-63.

7. Rottenberg $\mathrm{H}$, Waring A, Rubin E. Tole rance and cross-tolerance in chronic alcoholics : reduced membrane binding of ethano and other drugs. Science $1981 ; 213$ : 583-4.

8. Beaugé F, Fleuret C, Barin F, Nordmann $R$. Brain membrane disordering after acute in vivo administration of ethanol, isopropano or t-butanol in rats. Biochem Pharmacol $1984 ; 33: 3591-5$.

9. Hansson E, Gustavsson L, Jonsson G Alling C. Effect of ethanol on the lipid com position of astroglial cell culture. Alcohol Alcoholism 1986; 254 : A72 (abstr.)

10. Sun SH, Fu YH, Jou TC, Sun GY, Sun AY. Ethanol effects on phospholipids of rat brain astrocyte cell line. Alcohol Alcoholism 1986 ; 262 : A74 (abstr.).

11. Guerri C, Wallace R, Grisolia S. The influence of prolonged ethanol intake on the levels and turnover of alcohol and aldehyde dehydrogenase and of brain $\left(\mathrm{Na}^{+}, \mathrm{K}^{+}\right)$ATPase of rats. Eur J Biochem 1978; 63 : 323-9.

12. Ledig $\mathbf{M}$, Kopp $\mathbf{P}$, Mandel P. Effect o ethanol on adenosine triphosphatase and enolase activity in rat brain and in cultured nerve cells. Neurochem Res 1985 ; 10 : 131 124

13. Feiermann DE, Winston GW, Cederbaum AI. Ethanol oxidation by hydroxyl radicals role of iron chelates superoxide and hydrogen peroxide. Alcoholism: Clin Exp Res $1985 ; 9$ 95-101.

14. Ledig M, M'Paria JR, Mandel P. Super oxide dismutase activity in rat brain during acute and chronic ethanol intoxication. Neurochem Res 1981 ; 6 : 385-90.

15. Clemens $\mathrm{MR}$, Einsele $\mathrm{H}$, Remmer $\mathrm{H}$ Alcohol consumption and hepatic fibrosis affect the fatty acid composition of red blood cells and their susceptibility to lipid peroxi dation. Arch Toxicol 1987; 60 : 167-9.

16. Fuhrmann G, Besnard F, Kempf J, Kempf $E$, Ebel A. Influence of mouse genotype res ponses of central cholinergic neurotransmis sion to long term alcohol intoxication. Alcohol 1986 ; 3 : 291-8. tase (figure 2, page 355). Or, l'alcool peut induire une inhibition de l'activité de la superoxyde dismutase qui constitue une défense contre les multiples attaques des radicaux libres [14] (figure 3, page 355). Il en résulte une augmentation de radicaux superoxydes qui a pour effet une peroxydation des lipides membranaires avec une altération des membranes [15].

\section{Effets de l'alcool sur les neurotransmetteurs}

Le transfert des signaux et stimuli des sites récepteurs du système nerveux central aux sites réactionnels est assuré par les neurotransmetteurs. La recherche des effets de la consommation d'alcool sur ces neurotransmetteurs et leur mécanisme d'action soulèvent de nombreux problèmes fort complexes du fait des interrelations entre les divers types de neurones: cholinergiques, adrénergiques, sérotoninergiques, dopaminergiques, GABAergiques et peptidergiques. Étant donné que la répartition et la réponse des neurotransmetteurs peuvent varier selon les régions du cerveau, il convient d'étudier les effets de l'alcool dans des zones bien définies, anatomiquement et fonctionnellement. Là également nous allons retrouver des effets de sens opposés selon la zone cérébrale et la durée de l'alcoolisation.

L'acétylcholine. On considère que la réduction générale de l'activité du système nerveux central sous l'effet de l'alcool est pour une bonne part liée à une diminution de la libération d'acétylcholine. L'éthanol aurait, en effet, une action directe sur la transmission cholinergique en bloquant en partie la synthèse de l'acétylcholine. L'activité de la choline acétyltransférase (enzyme de synthèse de l'acétylcholine) est accélérée, en réaction sans doute à ce défaut de synthèse. Il en résulte des modifications de la disponibilité de l'acétylcholine, modifications dont l'importance varie selon la structure cérébrale considérée. Le striatum et l'hippocampe, sites d'interactions complexes entre des neurotransmetteurs majeurs - acétylcholine, dopamine, sérotonine, glutamate, acide $\gamma$ aminobutyrique (GABA) - seraient particulièrement affectés [ 16].
La noradrénaline. Dans l'ensemble, les effets de l'éthanol sur le niveau et le métabolisme de la noradrénaline apparaissent peu marqués. L'administration aiguë d'alcool n'affecte pas le taux de noradrénaline, mais pourrait agir sur sa recapture. La consommation d'alcool de longue durée (un an au moins chez le rat) entraîne une augmentation du taux cérébral de noradrénaline [17].

La sérotonine. L'effet aigu de l'alcool induit, selon la structure, une baisse ou une accélération du renouvellement de la sérotonine [18]. Quant au traitement chronique, les modifications qu'il entraîne varient en fonction de la durée du traitement. Les récepteurs sérotoninergiques ne semblent pas être affectés par la consommation d'alcool.

La dopamine. A la suite d'une intoxication aiguë, le fait dominant pour la dopamine paraît être une diminution de sa libération. Cette réduction de la décharge en transmetteur des neurones dopaminergiques est accompagnée d'une accélération de leur métabolisme, reflet probable d'une réaction de compensation à l'inhibition de la libération du transmetteur. Après consommation chronique, le phénomène de compensation s'épuise et le métabolisme de la dopamine baisse. L'inhibition de la libération de dopamine à partir des terminaisons nerveuses dans le striatum par exemple et le ralentissement de son métabolisme sont sans doute impliqués dans les incoordinations motrices que l'on observe après ingestion aiguë ou chronique d'alcool.

On sait par ailleurs que certaines amines biogènes se condensent avec l'acétaldéhyde pour former des composés ayant une structure voisine de la morphine [19]. Étant donné l'inhibition de l'acétaldéhyde déshydrogénase par l'alcool signalée chez les sujets alcoolodépendants, on peut envisager une accumulation d'acétaldéhyde disponible pour une telle condensation.

Tout récemment, la formation de complexes de l'acétaldéhyde avec des protéines (enzymes, hémoglobine, albumine) a été démontrée. Les anticorps dirigés contre ces substances pourraient constituer de nouveaux 


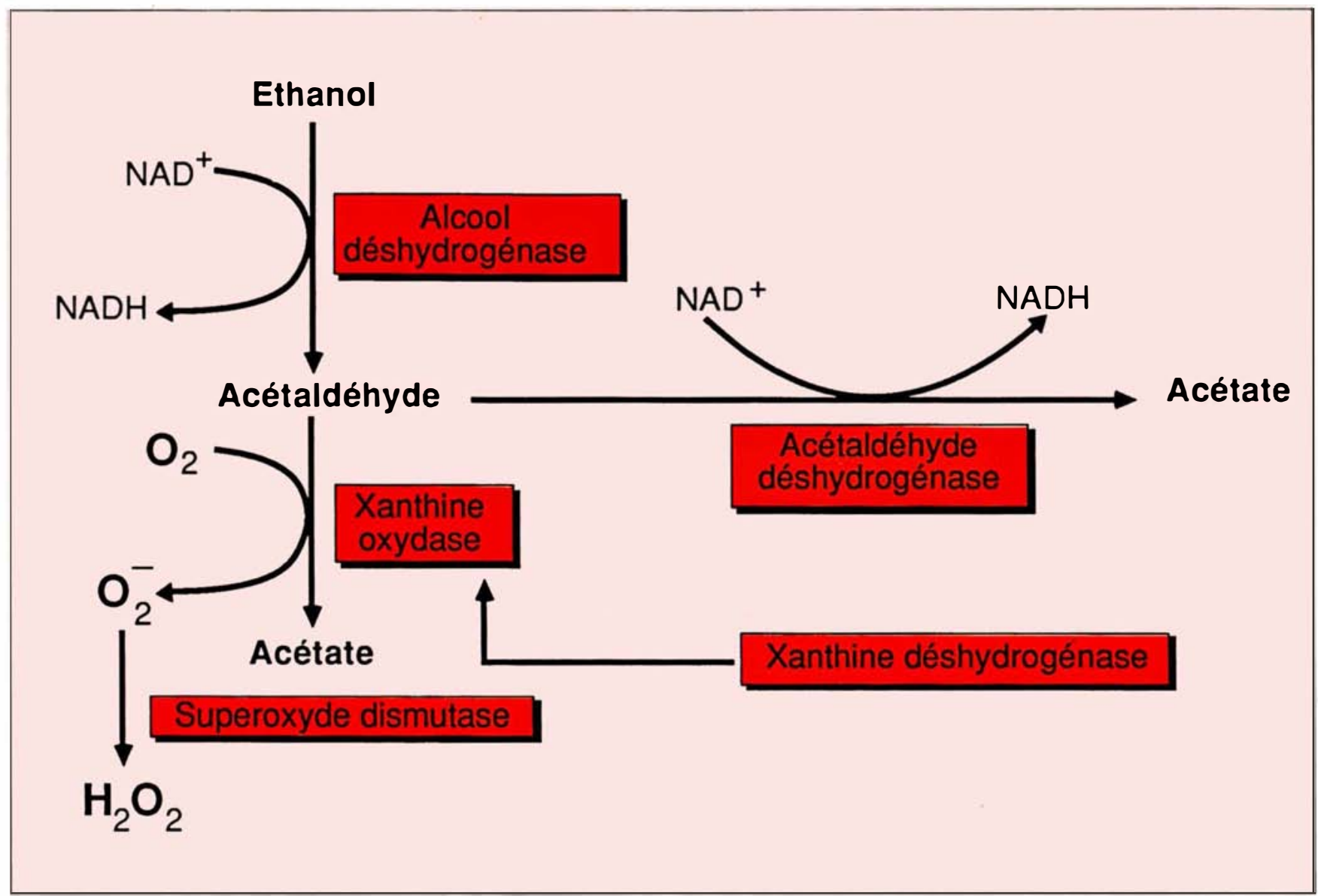

Figure 2. Schéma représentant les mécanismes de la dégradation de l'alcool.

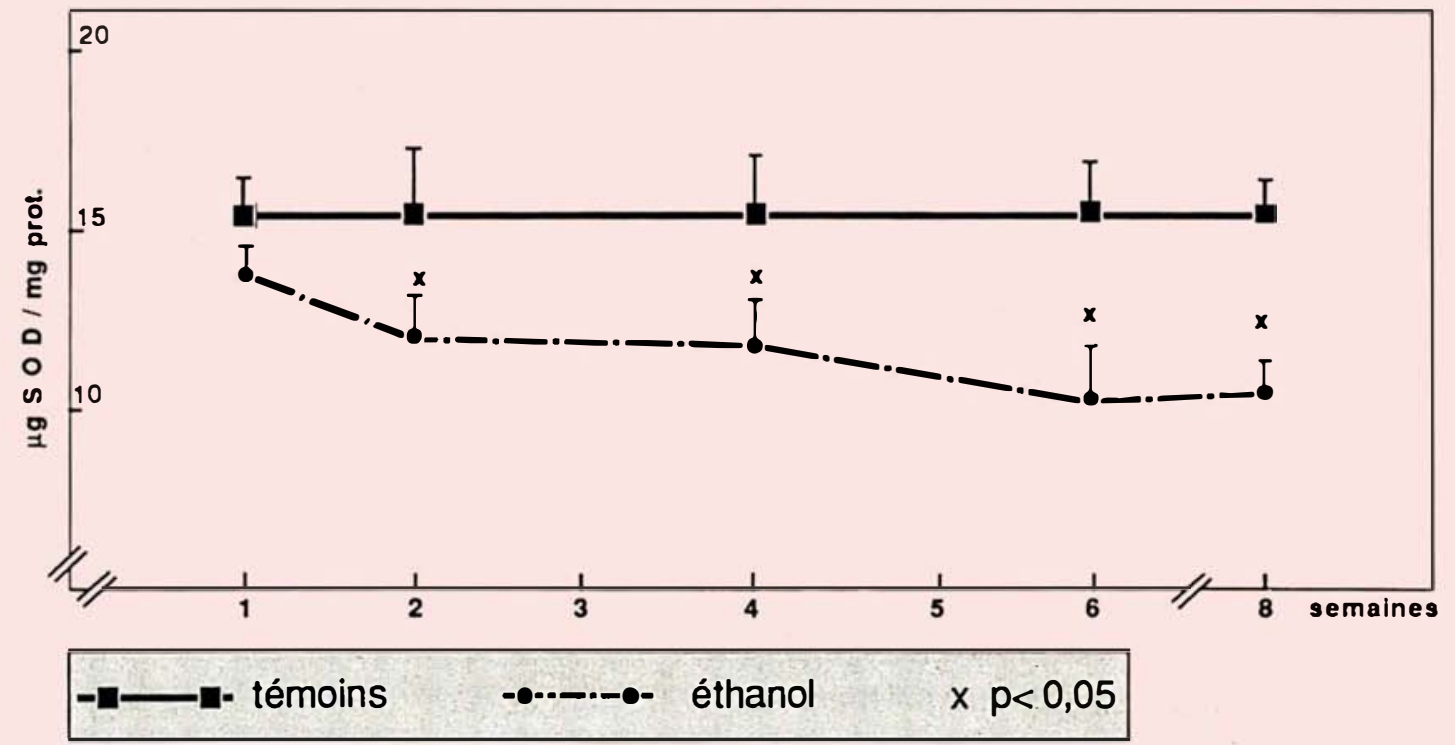

Figure 3. Activité de la superoxyde dismutase (SOD) de cerveau de rats témoins (D) et d'animaux ayant consommé de l'alcool à $20 \%(\bullet)$. $p<0,05$. A la suite de la baisse du rapport NAD+/NADH, la xanthine déshydrogénase se transforme en xanthine oxydase, entrainant l'apparition de radicaux superoxyde et une oxydation de l'acétaldéhyde en acétate. 


\section{RÉFÉRENCES}

17. Tewari S, Sytinsky IA. Alcohol. In : Lajtha A, ed. Handbook of Neurochemistry. New York: Plenum Press, 1984 : 219-61.

18. Besnard F, Kempf E, Fuhrmann G, Kempf J, Ebel A. Influence of mouse genotype on responses of central biogenic amines to alcohol intoxication and aging. Alcohol $1986 ; 3: 345-50$

19. Cohen G. Some neuropharmacologic properties of tetrahydroisoquinolines derived from the condensation of aldehyde with catecholamines. Finn Found Alcohol Stud 1975 ; 23 : $187-95$

20. Israel $\mathrm{Y}$, Arnon $\mathrm{R}$, Hurwitz E, Waks $\mathrm{T}$ Macdonald A. Monoclonal and polyclonal antibodies against acetaldehyde containing epitopes in modified proteins. Alcohol Alcoholism 1986; 20 : A7 (abstr.).

21. Davis VE, Walsh MS. In : Israel Y, Mardones J, eds. Biological Basis of Alcoholism. New York, Toronto: Wiley-Interscience, $1971: 73-102$

22. Simler S, Clement J, Ciesielski L, Mandel $P$. Brain $\gamma$-aminobutyric acid turnover rates after spontaneous chronic ethanol intake and withdrawal in discrete brain areas of C57 mice. J. Neurochem 1986; 47 : 1942-7.

23. Chan AWK. Alcoholism and Epilepsy. Epilepsia 1985; 26 : 323-33.

24. Suzdak PD, Paul SM. Ethanol membranes and neurotransmitters: novel approaches to modifying the behavioral actions of alcohol. Psychopharmacol Bull $1987 ; 23: 445-51$.

25. Ledig M, M'Paria JR, Mandel P. Free amino acids in the brain of ethanol treated rats. Subst Alcohol Actions Misuse 1982; 3 : 25-30.

26. Michaelis ML, Michaelis EK, Nunley EW, Galton N. Effect of chronic alcohol administration on synaptic membrane $\mathrm{Na}^{+}$. $\mathrm{Ca}^{2+}$ exchange activity. Brain Res $1987 ; 414$ : 239-44.

27. Carlen PL, Wilkinson A. Reversibility of alcohol-related brain damage: clinical and experimental observations. Acta Med Scand 1987 ; 717 (suppl.) : 19-26.

28. Kosterlitz H, Hughes J. Opiate receptors and endogenous opioid peptides in tolerance and dependance. In : Gross MM, ed. Alcohol Intoxication and Withdrawal. Vol. III B. New York: Plenum Press, 1977 : 141-54.

29. Myers RD, Critcher C. Naloxone alters alcohol drinking induced in the rat by tetrahydropapaveroline (THP) infused ICV. Pharmacol Biochem Behav 1982; 16 : 827-36.

30. Topel H. Biochemical basis of alcoholism. Staternent and hypotheses of present marqueurs de l'alcoolisme [20]. Ces observations se rapprochent de la théorie d'analogues de la morphine proposée par Davis et Walsh [21] mais dont la réalité physiologique n'a pas pu être mise en évidence à ce jour.

L'acide $\boldsymbol{\gamma}$-aminobutyrique (GABA). Le GABA est le neurotransmetteur inhibiteur majeur. Il intervient dans le contrôle de l'action des autres neurotransmetteurs. Son taux est abaissé dans de nombreuses affections (maladie de Parkinson, maladie d'Alzheimer, schizophrénie). L'alcool agit à la fois sur le taux, le métabolisme et les récepteurs du GABA. Lors de l'alcoolisation aiguë, sa teneur cellulaire aug. mente, parallèlement à un accroissement de la concentration en acide glutamique, sans doute à la suite du bloquage du cycle de Krebs. Lors de l'intoxication chronique le taux de synthèse du GABA est abaissé dans de nombreuses zones du cerveau [22]. L'alcool, étant un dépresseur du système nerveux central, peut potentialiser les effets du GABA. Nous avons constaté que, chez des rats nés de mères ayant consommé de l'alcool pendant la gestation et la période d'allaitement, le taux de GABA cérébral était modifié dans de nombreuses zones. Il est particulièrement augmenté dans l'amygdale. Dans certaines souches de souris ou de rats, le nombre de sites récepteurs du GABA baisse lors de l'intoxication alcoolique chronique. La réduction de la transmission GABAergique peut expliquer l'hyperexcitabilité produite par l'alcool. L'effet aggravant de la consommation d'alcool chez des malades épileptiques a fait l'objet de nombreux travaux [23]. On admet généralement qu'il est lié à une détérioration de la neurotransmission GABAergique. Les récepteurs des benzodiazépines voisins de ceux du GABA, sont également affectés, même par de faibles concentrations d'alcool [24].

L'acide glutamique. Contrairement au GABA, l'acide glutamique joue le rôle d'un neurotransmetteur excitateur. Nous avons observé une augmentation très significative de la teneur en acide glutamique cérébral chez des rats après une injection unique d'alcool de même qu'après un traitement chronique. L'alcool ne semble pas produire d'effet biphasique sur la concentration de l'acide glutamique [25].

D'une manière générale, on ne peut admettre une action ponctuelle de l'alcool sur l'un ou l'autre des neurotransmetteurs. Selon la dose d'alcool ingérée, la nature du neurotransmetteur ou de la structure centrale considérée, l'effet de l'éthanol sera plus ou moins accusé. Il s'ensuivra une rupture de l'équilibre de leurs interactions au sein des différentes structures cérébrales qui entraîneront les perturbations neurosensorielles et comportementales que l'on connaît chez l'alcoolique.

\section{Effet de l'alcool sur les canaux ioniques}

Le calcium joue un rôle essentiel dans la stabilité membranaire et dans la neurotransmission. L'alcoolisation aiguë entraîne une diminution et le traitement chronique une augmentation du taux de $\mathrm{Ca}^{++}$ membranaire [26]. Il a été suggéré que la capacité de transport du $\mathrm{Ca}^{++}$, variable selon la souche, pourrait expliquer la préférence pour l'alcool des souris C57 comparées aux souris DBA.

On sait par ailleurs que les canaux ioniques sont couplés aux neurorécepteurs. La fixation du ligand à son récepteur modifie la perméabilité du canal ionique. Un des systèmes les mieux étudiés est celui de la transmission catécholaminergique: il comporte un couplage adényl cyclase-AMP cyclique-protéine kinase. L'action aiguë de l'alcool, en modifiant la fluidité membranaire et le flux calcique, affecte le bon fonctionnement de ce système de neurotransmission. Quant aux canaux chloriques, ils sont en relation avec les récepteurs GABA et semblent être contrôlés par une protéine particulière, la GABA-moduline. L'action de l'alcool sur le flux ionique a été analysée chez l'animal au moyen de techniques électropharmacologiques [27]. Il est apparu que, dans la zone CAl de l'hippocampe, de faibles doses d'alcool inhibaient l'activité neuronale à la suite d'une hyperpolarisation des membranes et d'une augmentation de la conductance potassique, 
ce qui correspond à une ouverture des canaux potassium. Un effet semblable a été obtenu avec des anesthésiques. Sur des coupes d'hippocampe provenant de rats traités pendant 20 semaines par de l'éthanol, puis sevrés pendant trois semaines, on a observé une diminution de la conductance potassique en même temps qu'une augmentation du $\mathrm{Ca}^{++}$intracellulaire.

\section{Effet de l'alcool sur les neuropeptides}

Découverts par Kosterlitz [28], les neuropeptides, à certains égards proches des neurotransmetteurs, interviennent dans la régulation de nombreuses activités fonctionnelles du système nerveux central et en particulier dans divers comportements. Parmi les neuropeptides, les endorphines et les enképhalines ont tout particulièrement retenu l'attention. Les enképhalines sont des pentapeptides dont il existe deux types : la méthionine-enképhaline et la leucine-enképhaline. Pour les endorphines on distingue trois types: $\alpha, \beta$ et $\gamma$; ce sont des peptides à chaîne plus longue (16 à 31 acides aminés). Toutes ces substances ont des récepteurs spécifiques interagissant avec un canal calcique et, selon la zone cérébrale considérée, avec la plupart des neurotransmetteurs.

L'alcool favorise l'intervention des enképhalines. Étant donné leurs rôles multiples: dans la douleur, la régulation de la faim et de la soif, le sommeil, le comportement émotionnel, l'apprentissage et la mémorisation, on conçoit que l'alcool puisse perturber l'ensemble de ces fonctions. Les mécanismes qui sous-tendent ces effets ne sont que partiellement élucidés. Il a cependant été montré que certaines modifications du métabolisme de la dopamine ne sont pas dues à un effet direct de l'alcool, mais à une intervention des systèmes enképhalinergiques. Là encore l'effet de l'alcool sur les membranes, donc sur les récepteurs qui y sont localisés, constitue probablement un des mécanismes responsables des modifications observées. Une corrélation entre le taux de Met-enképhaline et les quantités d'alcool consommé $\mathrm{m} / \mathrm{s} n^{\circ} 6$ vol. 4, juin 88 par les souris C57 a été rapportée. Il est également intéressant de signaler que l'action des endorphines est inhibée par des antagonistes spécifiques de la morphine, comme la naloxone qui supprime les convulsions du syndrome de sevrage chez l'animal [29].

\section{Conclusion}

La consommation aiguë et/ou chronique d'alcool produit des altérations multiples, structurales et fonctionnelles, au niveau du cerveau. Il en résulte des malformations fœetales, des troubles des fonctions sensitives, sensorielles et motrices. Les cibles principales sont les membranes, les canaux ioniques et l'environnement moléculaire des récepteurs, ainsi que les réactions métaboliques auxquelles participe une coenzyme d'une importance capitale: le $\mathrm{NAD}^{+}$. L'administration aiguë d'alcool provoquant l'ivresse augmente la fluidité membranaire, entraînant une activation de certains neurotransmetteurs et une modification du flux ionique. En revanche, le traitement chronique et le sevrage entraînent un état de rigidité membranaire qui se traduirait par des phénomènes de dépendance. Ceci explique les effets biphasiques, en particulier en ce qui concerne les enzymes membranaires, les neuro-transmetteurs, les canaux ioniques, la densité et l'affinité des récepteurs liés à l'action des neuromédiateurs.

Quant à la neurotoxicité de l'alcool, elle semble être due à la fois à l'accumulation d'acétaldéhyde et à des radicaux libres qui sont à l'origine des altérations membranaires.

Les voies de recherche les plus prometteuses semblent être une extension des connaissances sur les composantes génétiques qui favorisent l'alcoolisme, sur les modifications des structures membranaires, et sur les protéines qui participent au métabolisme et à la réception du message des neurotransmetteurs. Une meilleure analyse de la dépendance liée sans doute aux systèmes endorphinergiques [30] pourrait peut-être conduire à une thérapeutique intéressante de l'alcoolisme

\section{Summary}

The effect of alcohol on nervous tissue was only studied recently. The main difficulty was to find out an animal model for experimentation, since normally animals avoid to drink alcohol. One of the model used to test the direct effect of alcohol on nervous tissue are cultured nerve cells. Other difficulties are the heterogeneity of nervous tissue as well as the genetic and individual variations in response to alcohol exposure. Acute and/or chronic alcohol consumption produce multiple structural and functional alterations of the nervous tissue as well as the fetal alcohol syndrome does, if alcohol is consumed during pregnancy. The main targets for modifications following alcohol consumption are membranes, ion channels and neurotransmitter metabolic pathways and receptors. Acute treatment increases membrane fluidity while chronic absorption and withdrawal produce membrane rigidity which may explain the biphasic effect of alcohol. Those events may also be involved in the disfunction of ion channels and neurotransmitter receptors. A better knowledge about the involvement of genetic factors would be necessary. There is some evidence that neuropeptides are involved in alcohol effect on nervous tissue. Here again, further studies would provide some basis for a drug therapy of alcoholism.

\section{TIRÉS A PART}

M. Ledig : centre de neurochimie du Cnrs, 5, rue Blaise-Pascal, 67084 Strasbourg Cedex, France 